\title{
Asymptotic analysis of Bragg fibers and dielectric coaxial fibers
}

\section{Yong Xu, Amnon Yariv}

Yong Xu, Amnon Yariv, "Asymptotic analysis of Bragg fibers and dielectric coaxial fibers," Proc. SPIE 4532, Active and Passive Optical Components for WDM Communication, (30 July 2001); doi: 10.1117/12.436012

SDIE Event: ITCom 2001: International Symposium on the Convergence of IT and Communications, 2001, Denver, CO, United States 


\title{
Asymptotic analysis of Bragg fibers and dielectric coaxial fibers
}

\author{
Yong Xu, Amnon Yariv \\ Department of Applied Physics, MS 128-95, \\ California Institute of Technology, Pasadena, CA 91125, USA
}

\begin{abstract}
Using an asymptotic matrix formalism, we analyze the guided modes of Bragg fibers and the dielectric coaxial fibers. In the asymptotic limit, the Bloch theorem can be applied to describe the optical field within the cladding layers, while the core region field is described by the exact solutions of Maxwell equations. From the asymptotic analysis, we derive an approximate expression for the radiation loss of Bragg fibers and dielectric coaxial fibers and give the number of Bragg pairs required to achieve $0.2 \mathrm{~dB} / \mathrm{km}$ radiation loss. The dispersions of the guided modes of Bragg fibers and dielectric coaxial fibers are calculated using both the asymptotic approach and the finite difference time domain method. The results obtained from these two approaches are shown to have excellent agreement. We use asymptotic analysis to calculate the dispersion parameter D of the guided dielectric coaxial fiber modes, which is found to be much larger than that of the conventional telecom fibers.
\end{abstract}

Keywords: Bragg Fibers, Dielectric Coaxial Fibers, Asymptotic Analysis, Fiber Dispersion

\section{INTRODUCTION}

In conventional optical fibers, the light confinement is achieved through total internal reflection and photons propagate mainly in the high index silica core. A completely different confinement mechanism, Bragg reflection, provides an alternative way of guiding photons, and has recently attracted a lot of attention. ${ }^{1-8}$ Since Bragg reflection and total internal reflection are completely different guiding mechanisms, it is not surprising that fibers with Bragg confinement offer many possibilities that are difficult to achieve in conventional fibers. A particularly appealing application of Bragg confinement is the possibility of guiding light in air instead of silica glass, which can lead to lower propagation loss and reduce the threshold for nonlinear effects. We can also utilize Bragg reflection to design a fiber that supports a single guided mode without azimuthal dependence. In contrast with the fundamental mode in conventional fibers, which is always doubly degenerate, these guided fiber modes are truly single mode. Consequently, many undesirable polarization dependent effects, such as polarization mode dispersion (PMD) and polarization dependent loss (PDL), can be completely eliminated. ${ }^{7}$

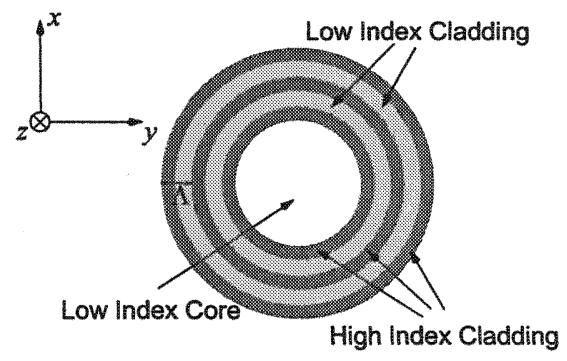

(a) Bragg Fiber

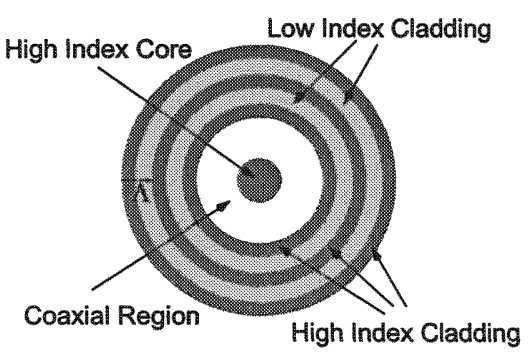

(b) Dielectric Coaxial Fiber

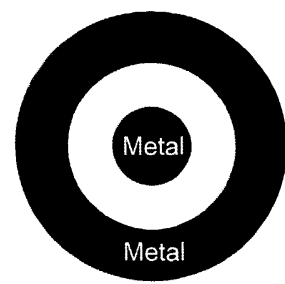

(c) Metallic Coaxial Cable

Figure 1. Schematic of (a) a Bragg fiber, (b) a dielectric coaxial fiber, and (c) a metallic coaxial cable.

The possibility of guiding light using Bragg confinement was first pointed out by Yeh et al., ${ }^{1}$ where the concept of Bragg fibers was proposed. The experimental fabrication of Bragg fibers has been recently reported. ${ }^{4}$ Fig. (1a) is the schematic of a Bragg fiber, which consists of a low index dielectric core surrounded by cladding layers with alternating high and low refractive indices. A new approach of using Bragg reflection to transmit optical signals

Y. Xu's E-mail address: yong@its.caltech.edu 
was recently syggested by Ibanescu et al. ${ }^{7}$ In this design, they proposed to use an all-dielectric coaxial fiber to overcome problems of polarization rotation and pulse broadening in high data rate telecommunication. The coaxial fiber is essentially a Bragg fiber with an extra high index core, as shown in Fig. (1b). The cladding of the coaxial fiber is a cylindrical omnidirectional mirror, which can be designed such that there is a frequency range within which light incident from the low index medium is completely reflected back irrespective of the incident angle and polarization. ${ }^{9 \cdot 11}$ Thus analogy can be drawn between dielectric coaxial fibers and metallic coaxial cables [see Fig. (1b) and Fig. (1c)]. Based on this analogy, Ibanescu et al. predicted small dispersion for dielectric coaxial fibers.

To find the dispersion and field distribution of the guided modes in Bragg fibers and coaxial fibers, we can use a two-dimensional (2D) finite difference time domain (FDTD) algorithm. ${ }^{12}$ The advantage of this approach is the ability to analyze fibers with arbitrary $2 \mathrm{D}$ profiles. The drawback is that the numerical approach tends to be time consuming and physically less transparent. Here we develop an efficient analytical method for Bragg fibers and coaxial fibers by taking advantage of their cylindrical symmetry and radial periodicity of the cladding layers.

\section{ASYMPTOTIC MATRIX THEORY}

We begin the asymptotic analysis by separating the cylindrical fibers into two regions: the core region and the cladding region. The core region consists of $\mathrm{N}$ concentric layers each with refractive index $n_{c o}^{i}$ and thickness $l_{c o}^{i}$, $i=1 \cdots N$. The cladding region is composed of pairs of alternating layers of different dielectric media. Layer type I has refractive index $n_{c l}^{1}$ and thickness $l_{c l}^{1}$. Layer type II has refractive index $n_{c l}^{2}$ and thickness $l_{c l}^{2}$, as shown in Fig. 2.

\subsection{Exact Solution in the Core Region}

In the asymptotic matrix formalism, we apply exact solutions of Maxwell equations to describe the fields in the core region. It should be emphasized that the refractive index and thickness of layers in the core region can be chosen arbitrarily. If we take the $z$ axis as the direction of propagation, due to the translational symmetry, every field component has the following form ${ }^{1}$

$$
\psi(r, \theta, z, t)=\psi(r, \theta) e^{i(\beta z-\omega t)},
$$

where $\psi$ can be $E_{z}, E_{r}, E_{\theta}, H_{z}, H_{r}$, and $H_{\theta}, \omega$ is the mode frequency, and $\beta$ is the propagation constant.

Due to the cylindrical symmetry of Bragg fibers, we can take the azimuthal dependence of the field components as $\cos (m \theta)$ or $\sin (m \theta)$. As a result, the electromagnetic field at radius $r$, which is within the $i$ th core layer, can be written in the following matrix form ${ }^{1}$

$$
\left[\begin{array}{c}
E_{z} \\
\frac{1}{i \beta} H_{\theta} \\
H_{z} \\
-\frac{1}{i \beta} E_{\theta}
\end{array}\right]=M\left(n_{c o}^{i}, k_{c o}^{i}, r\right)\left[\begin{array}{c}
A_{i} \\
B_{i} \\
C_{i} \\
D_{i}
\end{array}\right],
$$

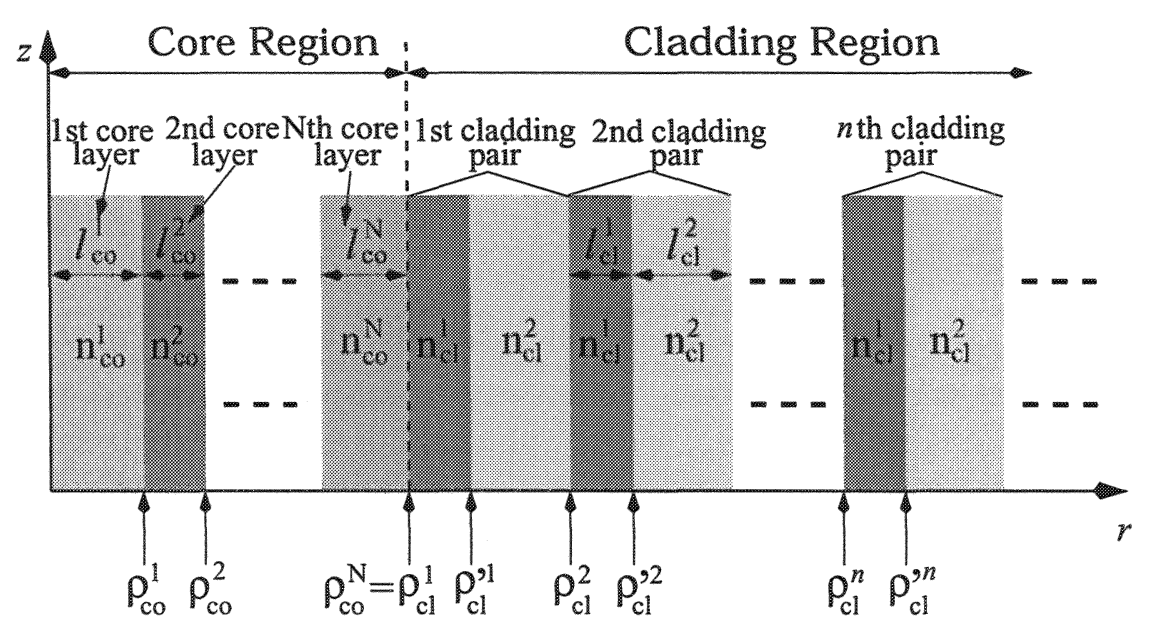

Figure 2. Schematic of the $r-z$ cross-section of a fiber with Bragg cladding. 
where the matrix $M\left(n_{c o}^{i}, k_{c o}^{i}, r\right)$ is defined as

$$
\begin{aligned}
M\left(n_{c o}^{i}, k_{c o}^{i}, r\right) & {\left[\begin{array}{cccc}
J_{m}\left(k_{c o}^{i} r\right) & Y_{m}\left(k_{c o}^{i} r\right) & 0 & 0 \\
\frac{\omega \epsilon_{0}\left(n_{c o}^{i}\right)^{2}}{k_{c o}^{i} \beta} J_{m}^{\prime}\left(k_{c o}^{i} r\right) & \frac{\omega \epsilon_{0}\left(n_{c o}^{i}\right)^{2}}{k_{c o}^{i} \beta} Y_{m}^{\prime}\left(k_{c o}^{i} r\right) & \frac{m}{\left(k_{c o}^{i}\right)^{2} r} J_{m}\left(k_{c o}^{i} r\right) & \frac{m}{\left(k_{c o}^{i}\right)^{2} r} Y_{m}\left(k_{c o}^{i} r\right) \\
0 & 0 & J_{m}\left(k_{c o}^{i} r\right) & Y_{m}\left(k_{c o}^{i} r\right) \\
\frac{m}{\left(k_{c o}^{i}\right)^{2} r} J_{m}\left(k_{c o}^{i} r\right) & \frac{m}{\left(k_{c o}^{i}\right)^{2} r} Y_{m}\left(k_{c o}^{i} r\right) & \frac{\omega \mu_{0}}{k_{c o}^{i} \beta} J_{m}^{\prime}\left(k_{c o}^{i} r\right) & \frac{\omega \mu_{0}}{k_{c o}^{i} \beta} Y_{m}^{\prime}\left(k_{c o}^{i} r\right)
\end{array}\right], }
\end{aligned}
$$

with $k_{c o}^{i}=\sqrt{\left(n_{c o}^{i} \omega / c\right)^{2}-\beta^{2}}$. The coefficients $A_{i}, B_{i}, C_{i}$, and $D_{i}$ are constant within the $i$ th layer. We emphasize that as long as $\beta \leq n_{c o}^{i} \omega / c$, Eq. (2) and Eq. (3) are the exact solution of Maxwell equations. ${ }^{1}$

Once the electromagnetic field in the $i$ th layer is known, we can easily find the field in the $(i+1)$ th layer by applying the condition that $E_{z}, E_{\theta}, H_{z}, H_{\theta}$ are continuous at $r=\rho_{c o}^{i}$, the interface between the $i$ th and $(i+1)$ th layer:

$$
\left[\begin{array}{c}
A_{i+1} \\
B_{i+1} \\
C_{i+1} \\
D_{i+1}
\end{array}\right]=\mathbf{T}_{\mathbf{i}}\left[\begin{array}{c}
A_{i} \\
B_{i} \\
C_{i} \\
D_{i}
\end{array}\right], \quad \mathbf{T}_{\mathbf{i}}=\left[M\left(n_{c o}^{i+1}, k_{c o}^{i+1}, \rho_{c o}^{i}\right)\right]^{-1} M\left(n_{c o}^{i}, k_{c o}^{i}, \rho_{c o}^{i}\right)
$$

We notice that within the first core layer, the coefficients $B_{1}$ and $D_{1}$ are zero, since $Y_{m}(x)$ is infinite at $x=0$.

\subsection{Asymptotic Approximation in the Cladding Region}

The electromagnetic fields in the cladding region can be described in the same way as the core regions fields. However, with a sufficiently large $r$, it suffices to approximate the exact solution in the asymptotic limit, which allows us to replace $J_{m}(x)$ and $Y_{m}(x)$ with $\exp (i x) / \sqrt{x}$ and $\exp (-i x) / \sqrt{x} \cdot{ }^{13}$ Under this condition, the field distribution in type I layer of the $n$th cladding pair can be written as: ${ }^{8}$

$$
\begin{cases}E_{z}=\frac{f_{T M}}{\sqrt{k_{c l}^{1} r}}\left[a_{n} e^{i k_{c l}^{1}\left(r-\rho_{c l}^{n}\right)}+b_{n} e^{-i k_{c l}^{1}\left(r-\rho_{c l}^{n}\right)}\right] & \\ H_{\theta}=-\frac{\omega \epsilon_{0}\left(n_{c l}^{1}\right)^{2}}{k_{c l}^{1}} \frac{f_{T M}}{\sqrt{k_{c l}^{1} r}}\left[a_{n} e^{i k_{c l}^{1}\left(r-\rho_{c l}^{n}\right)}-b_{n} e^{-i k_{c l}^{1}\left(r-\rho_{c l}^{n}\right)}\right] & \rho_{c l}^{n} \leq r<\rho_{c l}^{n}+l_{c l}^{1} . \\ H_{z}=\frac{f_{T E}}{\sqrt{k_{c l}^{1} r}}\left[c_{n} e^{i k_{c l}^{1}\left(r-\rho_{c l}^{n}\right)}+d_{n} e^{-i k_{c l}^{1}\left(r-\rho_{c l}^{n}\right)}\right] \\ E_{\theta}=\frac{\omega \mu_{0}}{k_{c l}^{1}} \frac{f_{T E}}{\sqrt{k_{c l}^{1} r}}\left[c_{n} e^{i k_{c l}^{1}\left(r-\rho_{c l}^{n}\right)}-d_{n} e^{-i k_{c l}^{1}\left(r-\rho_{c l}^{n}\right)}\right]\end{cases}
$$

Similarly, fields inside type II of the same cladding pair are given by

$$
\left\{\begin{array}{l}
E_{z}=\frac{f_{T M}}{\sqrt{k_{c l}^{2}}}\left[a_{n}^{\prime} e^{i k_{c l}^{2}\left(r-\rho_{c l}^{\prime n}\right)}+b_{n}^{\prime} e^{-i k_{c l}^{2}\left(r-\rho_{c l}^{\prime n}\right)}\right] \\
H_{\theta}=-\frac{\omega \epsilon_{0}\left(n_{c l}^{2}\right)^{2}}{k_{c l}^{2}} \frac{f_{T M}}{\sqrt{k_{c l}^{2} r}}\left[a_{n}^{\prime} e^{i k_{c l}^{2}\left(r-\rho_{c l}^{\prime n}\right)}-b_{n}^{\prime} e^{-i k_{c l}^{2}\left(r-\rho_{c l}^{\prime n}\right)}\right] \\
H_{z}=\frac{f_{T E}}{\sqrt{k_{c l}^{2}}}\left[c_{n}^{\prime} e^{i k_{c l}^{2}\left(r-\rho_{c l}^{\prime n}\right)}+d_{n}^{\prime} e^{-i k_{c l}^{2}\left(r-\rho_{c l}^{\prime n}\right)}\right] \\
E_{\theta}=\frac{\omega \mu_{0}}{k_{c l}^{2}} \frac{f_{T E}}{\sqrt{k_{c l}^{2} r}}\left[c_{n}^{\prime} e^{i k_{c l}^{2}\left(r-\rho_{c l}^{\prime n}\right)}-d_{n}^{\prime} e^{-i k_{c l}^{2}\left(r-\rho_{c l}^{\prime n}\right)}\right]
\end{array} \quad \rho_{c l}^{\prime n} \leq r<\rho_{c l}^{\prime n}+l_{c l}^{2} .\right.
$$

In Eq. (5) and (6), $k_{c l}^{1}=\sqrt{\left(n_{c l}^{1} \omega / c\right)^{2}-\beta^{2}}, k_{c l}^{2}=\sqrt{\left(n_{c l}^{2} \omega / c\right)^{2}-\beta^{2}}, \rho_{c l}^{n}, \rho_{c l}^{\prime n}$ are defined in Fig. 2. It should be noted that TM component (including $E_{z}$ and $H_{\theta}$ ) and TE component (including $H_{z}$ and $E_{\theta}$ ) are decoupled in the asymptotic limit, with TM component amplitude being $f_{T M}$ and TE component amplitude being $f_{T E}$. The values of $f_{T E}$ and $f_{T M}$ are constant within the entire Bragg cladding region.

The field amplitudes in type II layer of the $n$th cladding pair can be easily related to those in type I layer of the same cladding pair. By applying the condition of $E_{z}, E_{\theta}, H_{z}, H_{\theta}$ being continuous at $r=\rho_{c l}^{\prime n}$, we find

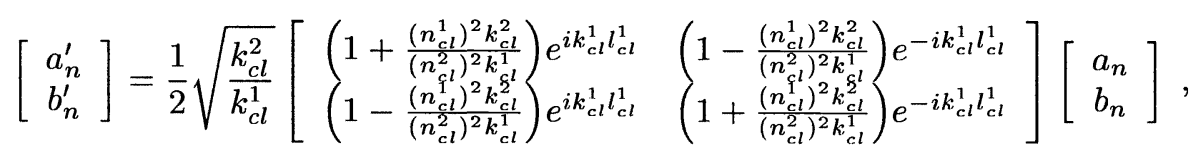


for the TM component, and

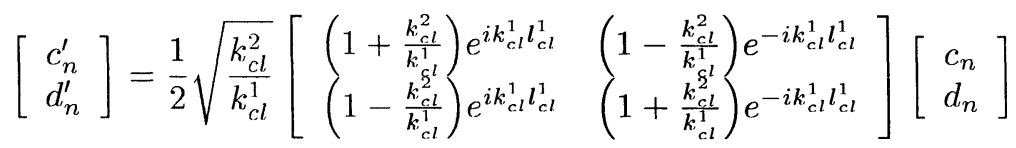

for the TE component. Similarly, we can relate $a_{n}^{\prime}, b_{n}^{\prime}, c_{n}^{\prime}$ and $d_{n}^{\prime}$ to $a_{n+1}, b_{n+1}, c_{n+1}$, and $d_{n+1}$ by applying the field continuity condition at $r=\rho_{c l}^{n+1}$. This allows us to express $a_{n+1}, b_{n+1}, c_{n+1}$ and $d_{n+1}$ in terms of the corresponding parameters in the $n$th layer:

$$
\begin{aligned}
& {\left[\begin{array}{l}
a_{n+1} \\
b_{n+1}
\end{array}\right]=\left[\begin{array}{ll}
A_{T M} & B_{T M} \\
B_{T M}^{*} & A_{T M}^{*}
\end{array}\right]\left[\begin{array}{l}
a_{n} \\
b_{n}
\end{array}\right],} \\
& {\left[\begin{array}{l}
c_{n+1} \\
d_{n+1}
\end{array}\right]=\left[\begin{array}{ll}
A_{T E} & B_{T E} \\
B_{T E}^{*} & A_{T E}^{*}
\end{array}\right]\left[\begin{array}{l}
c_{n} \\
d_{n}
\end{array}\right] .}
\end{aligned}
$$

The parameters $A_{T E}, B_{T E}, A_{T M}$ and $B_{T M}$ are respectively defined as:

$$
\begin{aligned}
& A_{T E}=e^{i k_{c l}^{1} l_{c l}^{1}}\left[i \frac{\left(k_{c l}^{1}\right)^{2}+\left(k_{c l}^{2}\right)^{2}}{2 k_{c l}^{1} k_{c l}^{2}} \sin \left(k_{c l}^{2} l_{c l}^{2}\right)+\cos \left(k_{c l}^{2} l_{c l}^{2}\right)\right], \\
& B_{T E}=i e^{-i k_{c l}^{1} l_{c l}^{1}} \frac{\left(k_{c l}^{1}\right)^{2}-\left(k_{c l}^{2}\right)^{2}}{2 k_{c l}^{1} k_{c l}^{2}} \sin \left(k_{c l}^{2} l_{c l}^{2}\right), \\
& A_{T M}=e^{i k_{c l}^{1} l_{c l}^{1}}\left[i \frac{\left(n_{c l}^{2}\right)^{4}\left(k_{c l}^{1}\right)^{2}+\left(n_{c l}^{1}\right)^{4}\left(k_{c l}^{2}\right)^{2}}{2\left(n_{c l}^{1}\right)^{2}\left(n_{c l}^{2}\right)^{2} k_{c l}^{1} k_{c l}^{2}} \sin \left(k_{c l}^{2} l_{c l}^{2}\right)+\cos \left(k_{c l}^{2} l_{c l}^{2}\right)\right], \\
& B_{T M}=i e^{-i k_{c l}^{1} l_{c l}^{1}} \frac{\left(n_{c l}^{2}\right)^{4}\left(k_{c l}^{1}\right)^{2}-\left(n_{c l}^{1}\right)^{4}\left(k_{c l}^{2}\right)^{2}}{2\left(n_{c l}^{1}\right)^{2}\left(n_{c l}^{2}\right)^{2} k_{c l}^{1} k_{c l}^{2}} \sin \left(k_{c l}^{2} l_{c l}^{2}\right) .
\end{aligned}
$$

Since $A_{T E}, B_{T E}, A_{T M}$, and $B_{T M}$ are the same for all cladding layers, we can apply the Bloch theorem to the cladding fields:

$$
\begin{gathered}
{\left[\begin{array}{l}
a_{n} \\
b_{n}
\end{array}\right]=\left(\lambda_{T M}\right)^{n-1}\left[\begin{array}{c}
B_{T M} \\
\lambda_{T M}-A_{T M}
\end{array}\right], \lambda_{T M}=\operatorname{Re}\left(A_{T M}\right) \pm \sqrt{\left[\operatorname{Re}\left(A_{T M}\right)\right]^{2}-1}} \\
{\left[\begin{array}{c}
c_{n} \\
d_{n}
\end{array}\right]=\left(\lambda_{T E}\right)^{n-1}\left[\begin{array}{c}
B_{T E} \\
\lambda_{T E}-A_{T E}
\end{array}\right], \lambda_{T E}=\operatorname{Re}\left(A_{T E}\right) \pm \sqrt{\left[\operatorname{Re}\left(A_{T E}\right)\right]^{2}-1}}
\end{gathered}
$$

These results clearly indicate that in the asymptotic limit, the properties of Bragg fiber cladding resemble those of planar Bragg stacks, ${ }^{14}$ which is directly due to the radial periodicity of the cladding layers and the fact that the asymptotic solutions in Eq. (5) and (6) takes the form of traveling plane waves. There are two solutions for $\lambda_{T E}$ and $\lambda_{T M}$. In the Bragg bandgap, which is defined by the condition of $\operatorname{Re}\left(A_{T E}\right)>1$ or $\operatorname{Re}\left(A_{T M}\right)>1$, the two solutions of $\lambda_{T E}$ and $\lambda_{T M}$ are real numbers, with one having absolute value less than one and the other greater than one. We shall take the solutions of $\lambda_{T E}$ and $\lambda_{T M}$ with absolute values less than unity, since they correspond to modes decaying in the Bragg cladding. Once we find the values of $a_{n}, b_{n}, c_{n}, d_{n}, a_{n}^{\prime}, b_{n}^{\prime}, c_{n}^{\prime}, d_{n}^{\prime}$ from Eq. (15) and Eq. (16), the only unknown quantities in Eq. (5) and Eq. (6), which give the electromagnetic field in the entire cladding region, are $f_{T E}$ and $f_{T M}$. The problem of finding them lies at the center of our asymptotic matrix formalism.

\subsection{Matrix Formalism}

The guided modes in a Bragg fiber are founded by matching the exact solution in the core region [i.e., Eq. (2)] with the asymptotic solution in the cladding region [i.e., Eq. (5)] at the interface $r=\rho_{c o}^{N}=\rho_{c l}^{1}$, which gives us

$$
M\left(n_{c o}^{N}, k_{c o}^{N}, \rho_{c o}^{N}\right)\left[\begin{array}{c}
A_{N} \\
B_{N} \\
C_{N} \\
D_{N}
\end{array}\right]=\left[\begin{array}{c}
\frac{f_{T M}}{\sqrt{k_{c l}^{1} \rho_{c l}^{1}}}\left(\lambda_{T M}-A_{T M}+B_{T M}\right) \\
-\frac{i \omega \epsilon_{0}\left(n_{c l}^{1}\right)^{2}}{k_{c l}^{1} \beta} \frac{f_{T M}}{\sqrt{k_{c l}^{1} \rho_{c l}^{1}}}\left(\lambda_{T M}-A_{T M}-B_{T M}\right) \\
\frac{f_{T E}}{\sqrt{k_{c}^{1} \rho_{c l}^{1}}}\left(\lambda_{T E}-A_{T E}+B_{T E}\right) \\
-\frac{i \omega \mu_{0}}{k_{c l}^{1} \beta} \frac{f_{T E}}{\sqrt{k_{c l}^{1} \rho_{c l}^{1}}}\left(\lambda_{T E}-A_{T E}-B_{T E}\right)
\end{array}\right] .
$$


We then relate the amplitude coefficients in the $N$ th core layer (i.e., $A_{N}, B_{N}, C_{N}$ and $D_{N}$ ) to the coefficients in the first core layer (i.e., $A_{1}, B_{1}, C_{1}$ and $D_{1}$ ). We remember that in the first core layer $B_{1}=D_{1}=0$ and we further denote $A_{1}$ as $\mathcal{A}_{T M}$, and $C_{1}$ as $\mathcal{C}_{T E}$. Applying Eq. (4) repeatedly, we have

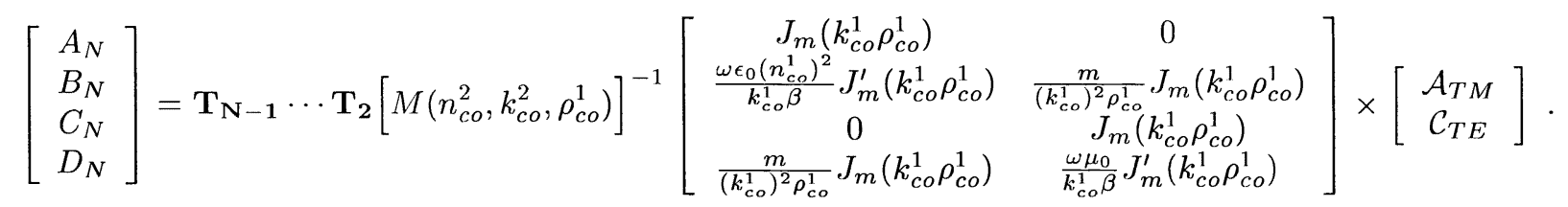

Substituting Eq. (18) into Eq. (17), we find the following matrix relation

$$
\left[\begin{array}{cc}
J_{m}\left(k_{c o}^{1} \rho_{c o}^{1}\right) & 0 \\
\frac{\omega \epsilon_{0}\left(n_{c o}^{1}\right)^{2}}{k_{c o}^{1} \beta} J_{m}^{\prime}\left(k_{c o}^{1} \rho_{c o}^{1}\right) & \frac{m}{\left(k_{c o}^{1}\right)^{2} \rho_{c o}^{1}} J_{m}\left(k_{c o}^{1} \rho_{c o}^{1}\right) \\
0 & J_{m}\left(k_{c o}^{1} \rho_{c o}^{1}\right) \\
\frac{m}{\left(k_{c o}^{1}\right)^{2} \rho_{c o}^{1}} J_{m}\left(k_{c o}^{1} \rho_{c o}^{1}\right) & \frac{\omega \mu_{0}}{k_{c o}^{1} \beta} J_{m}^{\prime}\left(k_{c o}^{1} \rho_{c o}^{1}\right)
\end{array}\right]\left[\begin{array}{c}
\mathcal{A}_{T M} \\
\mathcal{C}_{T E}
\end{array}\right]=\mathbf{T}\left[\begin{array}{c}
\frac{f_{T M}}{\sqrt{k_{c l}^{1} \rho_{c l}^{1}}}\left(\lambda_{T M}-A_{T M}+B_{T M}\right) \\
-\frac{i \omega \epsilon_{0}\left(n_{c l}^{1}\right)^{2}}{k_{c l}^{1} \beta} \frac{f_{T M}}{\sqrt{k_{c l}^{1} \rho_{c l}^{1}}}\left(\lambda_{T M}-A_{T M}-B_{T M}\right) \\
\frac{f_{T E}}{\sqrt{k_{c l}^{1} \rho_{c l}^{1}}}\left(\lambda_{T E}-A_{T E}+B_{T E}\right) \\
-\frac{i \omega \mu_{0}}{k_{c l}^{1} \beta} \frac{f_{T E}}{\sqrt{k_{c l}^{1} \rho_{c l}^{1}}}\left(\lambda_{T E}-A_{T E}-B_{T E}\right)
\end{array}\right]
$$

where an overall transfer matrix $\mathbf{T}$ is defined as

$$
\begin{aligned}
\mathbf{T} & =\left[M\left(n_{c o}^{2}, k_{c o}^{2}, \rho_{c o}^{1}\right) M^{-1}\left(n_{c o}^{2}, k_{c o}^{2}, \rho_{c o}^{2}\right)\right] \cdots\left[M\left(n_{c o}^{N}, k_{c o}^{N}, \rho_{c o}^{N-1}\right) M^{-1}\left(n_{c o}^{N}, k_{c o}^{N}, \rho_{c o}^{N}\right)\right] \\
& =\prod_{i=2}^{N}\left[M\left(n_{c o}^{i}, k_{c o}^{i}, \rho_{c o}^{i-1}\right) M^{-1}\left(n_{c o}^{i}, k_{c o}^{i}, \rho_{c o}^{i}\right)\right]=\left[\begin{array}{cccc}
t_{11} & t_{12} & t_{13} & t_{14} \\
t_{21} & t_{22} & t_{23} & t_{24} \\
t_{31} & t_{32} & t_{33} & t_{34} \\
t_{41} & t_{42} & t_{43} & t_{44}
\end{array}\right] .
\end{aligned}
$$

In Eq. (19), $\mathcal{A}_{T M}$ and $\mathcal{C}_{T E}$, which represent field in the first core layer, are linearly related to field in the first cladding layer $\left(f_{T M}\right.$ and $\left.f_{T E}\right)$ via a $4 \times 4$ transfer matrix $\mathbf{T}$ as defined in Eq. (20). Eq. (19) gives us four equations with four independent variables $\mathcal{A}_{T M}, \mathcal{C}_{T E}, f_{T M}, f_{T E}$, and is sufficient to determine the dispersion of the guided modes. To see this more clearly, we introduce eight new parameters $g_{T M}^{j}$ and $g_{T E}^{j}, j=1, \cdots, 4$ as

$$
\begin{gathered}
g_{T E}^{j}=t_{j 3}\left(\lambda_{T E}-A_{T E}+B_{T E}\right)-\frac{i \omega \mu_{0}}{k_{c l}^{1} \beta} t_{j 4}\left(\lambda_{T E}-A_{T E}-B_{T E}\right), j=1, \cdots, 4, \\
g_{T M}^{j}=t_{j 1}\left(\lambda_{T M}-A_{T M}+B_{T M}\right)-\frac{i \omega \epsilon_{0}\left(n_{c l}^{1}\right)^{2}}{k_{c l}^{1} \beta} t_{j 2}\left(\lambda_{T M}-A_{T M}-B_{T M}\right), j=1, \cdots, 4,
\end{gathered}
$$

where $t_{j 1}, j_{j 2}, t_{j 3}$ and $t_{j 4}$ are the matrix elements given in Eq. (20). With these new parameters, we can split Eq. (19) into two equations:

$$
\begin{gathered}
{\left[\begin{array}{cc}
J_{m}\left(k_{c o}^{1} \rho_{c o}^{1}\right) & 0 \\
\frac{\omega \epsilon_{0}\left(n_{c o}^{1}\right)^{2}}{k_{c o}^{1} \beta} J_{m}^{\prime}\left(k_{c o}^{1} \rho_{c o}^{1}\right) & \frac{m}{\left(k_{c o}^{1}\right)^{2} \rho_{c o}^{1}} J_{m}\left(k_{c o}^{1} \rho_{c o}^{1}\right)
\end{array}\right]\left[\begin{array}{c}
\mathcal{A}_{T M} \\
\mathcal{C}_{T E}
\end{array}\right]=\frac{1}{\sqrt{k_{c l}^{1} \rho_{c l}^{1}}}\left[\begin{array}{cc}
g_{T M}^{1} & g_{T E}^{1} \\
g_{T M}^{2} & g_{T E}^{2}
\end{array}\right]\left[\begin{array}{c}
f_{T M} \\
f_{T E}
\end{array}\right]} \\
\left.\begin{array}{cc}
0 & J_{m}\left(k_{c o}^{1} \rho_{c o}^{1}\right) \\
\frac{m}{\left(k_{c o}^{1}\right)^{2} \rho_{c o}^{1}} J_{m}\left(k_{c o}^{1} \rho_{c o}^{1}\right) & \frac{\omega \mu_{0}}{k_{c o}^{1} \beta} J_{m}^{\prime}\left(k_{c o}^{1} \rho_{c o}^{1}\right)
\end{array}\right]\left[\begin{array}{c}
\mathcal{A}_{T M} \\
\mathcal{C}_{T E}
\end{array}\right]=\frac{1}{\sqrt{k_{c l}^{1} \rho_{c l}^{1}}}\left[\begin{array}{cc}
g_{T M}^{3} & g_{T E}^{3} \\
g_{T M}^{4} & g_{T E}^{4}
\end{array}\right]\left[\begin{array}{c}
f_{T M} \\
f_{T E}
\end{array}\right] .
\end{gathered}
$$

These two equations lie at the center of our asymptotic matrix method. To fully understand their consequences, we consider two separate cases, the TE or TM modes with $m=0$, and the mixed modes with $m \neq 0$.

For modes with $m=0$, we first notice that the matrix $M\left(n_{c o}^{i}, k_{c o}^{i}, r\right)$ is block diagonalized into two $2 \times 2$ matrices. As a result, the transfer matrix T, as defined in Eq. (20), is also block diagonalized into two $2 \times 2$ matrices with $t_{31}=t_{41}=t_{32}=t_{42}=t_{13}=t_{23}=t_{14}=t_{24}=0$. According to the definitions in Eq. (21) and Eq. (22), we have $g_{T M}^{3}=g_{T M}^{4}=0$, and $g_{T E}^{1}=g_{T E}^{2}=0$.

By definition, the $H_{z}$ component of any TM mode must remain zero in the entire Bragg fiber, i.e., $\mathcal{C}_{T E}=0$ and $f_{T E}=0$. With this condition in mind, from Eq. (23) we can easily find

$$
\frac{\omega \epsilon_{0}\left(n_{c o}^{1}\right)^{2}}{k_{c o}^{1} \beta} \frac{J_{0}^{\prime}\left(k_{c o}^{1} \rho_{c o}^{1}\right)}{J_{0}\left(k_{c o}^{1} \rho_{c o}^{1}\right)}=\frac{g_{T M}^{2}}{g_{T M}^{1}} .
$$


Once we have specified the Bragg fiber parameters and chosen the frequency $\omega$, the propagation constants of TM modes are found by solving for $\beta_{T M}$ satisfying Eq. (25). We substitute the result $\beta_{T M}$ back into Eq. (23), and obtain the following relation

$$
\mathcal{A}_{T M}=\frac{g_{T M}^{1}}{J_{0}\left(k_{c o}^{1} \rho_{c o}^{1}\right) \sqrt{k_{c l}^{1} \rho_{c l}^{1}}} f_{T M} .
$$

The importance of this result is that it relates the mode amplitude $\mathcal{A}_{T M}$ in the first core layer to $f_{T M}$, which determines the fields within the entire fiber cladding region. We can choose the normalization factor of the guided mode such that $\mathcal{A}_{T M}=1$. Combining this condition with Eq. (26), $f_{T E}=0$, and the results in Sec. 2 , we obtain the TM field distribution in the cladding region. The TM field distribution in the core region can also be easily found. In the center core layer, we have $A_{1}=\mathcal{A}_{T M}=1$, and $B_{1}=C_{1}=D_{1}=0$. Applying Eq. (4) repeatedly, we find all the mode coefficients $A_{i}, B_{i}, C_{i}$, and $D_{i}$ in the $\mathrm{N}$ core layers. The TM field distribution in the core region is simply given by substituting these mode coefficients into Eq. (2) and applying Eq. (3).

For TE modes, we have $A_{T M}=0$ and Eq. (24) gives us

$$
\frac{\omega \mu_{0}}{k_{c o}^{1} \beta} \frac{J_{0}^{\prime}\left(k_{c o}^{1} \rho_{c o}^{1}\right)}{J_{0}\left(k_{c o}^{1} \rho_{c o}^{1}\right)}=\frac{g_{T E}^{4}}{g_{T E}^{3}}, \mathcal{C}_{T E}=\frac{g_{T E}^{3}}{J_{0}\left(k_{c o}^{1} \rho_{c o}^{1}\right) \sqrt{k_{c l}^{1} \rho_{c l}^{1}}} f_{T E}
$$

Following the same procedure as for the TM modes, we can find the propagation constant $\beta$ and field distribution for the TE modes.

For any mixed mode with $m \neq 0$, both Eq. (23) and Eq. (24) are needed and the solutions are more complicated. To simplify our final results, we introduce more definitions

$$
\begin{gathered}
H_{T E}^{1}=-J_{m}\left(k_{c o}^{1} \rho_{c o}^{1}\right) g_{T E}^{4}+\frac{\omega \mu_{0}}{k_{c o}^{1} \beta} J_{m}^{\prime}\left(k_{c o}^{1} \rho_{c o}^{1}\right) g_{T E}^{3}+\frac{m}{\left(k_{c o}^{1}\right)^{2} \rho_{c o}^{1}} J_{m}\left(k_{c o}^{1} \rho_{c o}^{1}\right) g_{T E}^{1}, \\
H_{T E}^{2}=J_{m}\left(k_{c o}^{1} \rho_{c o}^{1}\right) g_{T E}^{2}-\frac{\omega \epsilon_{0}\left(n_{c o}^{1}\right)^{2}}{k_{c o}^{1} \beta} J_{m}^{\prime}\left(k_{c o}^{1} \rho_{c o}^{1}\right) g_{T E}^{1}-\frac{m}{\left(k_{c o}^{1}\right)^{2} \rho_{c o}^{1}} J_{m}\left(k_{c o}^{1} \rho_{c o}^{1}\right) g_{T E}^{3}, \\
H_{T M}^{1}=J_{m}\left(k_{c o}^{1} \rho_{c o}^{1}\right) g_{T M}^{4}-\frac{\omega \mu_{0}}{k_{c o}^{1} \beta} J_{m}^{\prime}\left(k_{c o}^{1} \rho_{c o}^{1}\right) g_{T M}^{3}-\frac{m}{\left(k_{c o}^{1}\right)^{2} \rho_{c o}^{1}} J_{m}\left(k_{c o}^{1} \rho_{c o}^{1}\right) g_{T M}^{1}, \\
H_{T M}^{2}=-J_{m}\left(k_{c o}^{1} \rho_{c o}^{1}\right) g_{T M}^{2}+\frac{\omega \epsilon_{0}\left(n_{c o}^{1}\right)^{2}}{k_{c o}^{1} \beta} J_{m}^{\prime}\left(k_{c o}^{1} \rho_{c o}^{1}\right) g_{T M}^{1}+\frac{m}{\left(k_{c o}^{1}\right)^{2} \rho_{c o}^{1}} J_{m}\left(k_{c o}^{1} \rho_{c o}^{1}\right) g_{T M}^{3} .
\end{gathered}
$$

To find the propagation constant $\beta$ of any mixed mode, we first express $\mathcal{A}_{T M}$ and $\mathcal{C}_{T E}$ in terms of $f_{T M}$ and $f_{T E}$ by inverting the leftmost $2 \times 2$ matrix in Eq. (23). Substituting the results of $\mathcal{A}_{T M}$ and $\mathcal{C}_{T E}$ into Eq. (24), we find

$$
\left[\begin{array}{ll}
H_{T M}^{1} & -H_{T E}^{1} \\
H_{T M}^{2} & -H_{T E}^{2}
\end{array}\right]\left[\begin{array}{c}
f_{T M} \\
f_{T E}
\end{array}\right]=0
$$

with $H_{T E}^{1}, H_{T E}^{2}, H_{T M}^{1}$ and $H_{T M}^{2}$ defined in Eq. (28) through Eq. (31). In order for Eq. (32) to have non-zero solutions, the determinant of the matrix must be zero, which gives

$$
\frac{H_{T M}^{1}}{H_{T M}^{2}}=\frac{H_{T E}^{1}}{H_{T E}^{2}} .
$$

As can be seen from the definitions in Eq. (28) to Eq. (31), the parameters $H_{T E}^{1}, H_{T E}^{2}, H_{T M}^{1}$, and $H_{T M}^{2}$ are complicated. However, once the Bragg fiber structure is chosen and the frequency is given, they only depend on $\beta$. Therefore, the solution of Eq. (33) gives us the propagation constant of any mixed mode.

After finding the solutions of Eq. (33) and choosing an appropriate normalization constant, we can determine the values of $f_{T M}$ and $f_{T E}$ from Eq. (32):

$$
\left[\begin{array}{c}
f_{T M} \\
f_{T E}
\end{array}\right]=\frac{m}{\left(k_{c o}^{1}\right)^{2} \rho_{c o}^{1}}\left[J_{m}\left(k_{c o}^{1} \rho_{c o}^{1}\right)\right]^{2} \sqrt{k_{c l}^{1} \rho_{c l}^{1}}\left[\begin{array}{c}
H_{T E}^{1} \\
H_{T M}^{1}
\end{array}\right] .
$$


As before, by combining this result with those in Sec. 2, we can find the whole cladding field distribution. To obtain the fields in the fiber core region, we substitute Eq. (34) into Eq. (24) and find

$$
\left[\begin{array}{c}
\mathcal{A}_{T M} \\
\mathcal{C}_{T E}
\end{array}\right]=\left[\begin{array}{c}
-\frac{\omega \mu_{0}}{k_{c o}^{1} \beta} J_{m}^{\prime}\left(k_{c o}^{1} \rho_{c o}^{1}\right)\left(g_{T M}^{3} H_{T E}^{1}+g_{T E}^{3} H_{T M}^{1}\right)+J_{m}\left(k_{c o}^{1} \rho_{c o}^{1}\right)\left(g_{T M}^{4} H_{T E}^{1}+g_{T E}^{4} H_{T M}^{1}\right) \\
\frac{m}{\left(k_{c o}^{1}\right)^{2} \rho_{c o}^{1}} J_{m}\left(k_{c o}^{1} \rho_{c o}^{1}\right)\left(g_{T M}^{3} H_{T E}^{1}+g_{T E}^{3} H_{T M}^{1}\right)
\end{array}\right] .
$$

Thus within the first core layer, we have $A_{1}=\mathcal{A}_{T M}, C_{1}=\mathcal{C}_{T E}$, and $B_{1}=D_{1}=0$. By applying the equations in Sec. 2.1 throughout the entire core layers, we find the electromagnetic fields in the Bragg fiber core region.

\section{RADIATION LOSS}

Two sources contribute to the propagation loss in Bragg fibers or coaxial fibers: the material absorption loss and the radiation loss. The material absorption loss depends on the choice of dielectric medium. The radiation loss mainly depends on the index contrast between the cladding media and the number of cladding pairs. In principle, the radiation loss can be reduced below any given number simply by using a large enough number of cladding pairs. In this case, the propagation loss in an air core fiber is mostly due to the residual absorption in the fiber cladding. Thus if appropriate cladding materials can be found, it is possible to reduce the propagation loss in an air core fiber below that of conventional optical fibers. However, since using too many cladding pairs is generally undesirable or even impractical, it is important to know how many layers are required to reduce the radiation loss below a given number.

To simplify our analysis, we study a Bragg fiber with a central air core bounded by $\mathrm{N}$ pairs of cladding layers, as shown in Fig. 3. We treat the air core exactly and apply the asymptotic approximation to the entire Bragg cladding structure. This means that the transfer matrix $\mathbf{T}$ relating the cladding region to the core region is simply a $4 \times 4$ identity matrix. From Eq. (21) and Eq. (22), we find

$$
\begin{gathered}
g_{T E}^{3}=\lambda_{T E}-A_{T E}+B_{T E}, \\
g_{T E}^{4}=-\frac{i \omega \mu_{0}}{k_{c l}^{1} \beta}\left(\lambda_{T E}-A_{T E}-B_{T E}\right), \\
g_{T M}^{1}=\lambda_{T M}-A_{T M}+B_{T M}, \\
g_{T M}^{2}=-\frac{i \omega \epsilon_{0}\left(n_{c l}^{1}\right)^{2}}{k_{c l}^{1} \beta}\left(\lambda_{T M}-A_{T M}-B_{T M}\right) .
\end{gathered}
$$

Other values of $g_{T E}^{i}$ and $g_{T M}^{i}$ are all zero. As a further simplification, we shall confine ourselves to the study of $\mathrm{TE}$ and TM modes. The reasons are two-fold. As we have mentioned before, the modes with $m=0$ are of special interest, since they do not have any polarization dependent effects. Secondly, in the asymptotic limit, the mixed modes $(m \neq 0)$ in the cladding structure can always be classified into a TE component and a TM component, and should exhibit similar radiation loss characteristics.

We first consider TE modes. According to Eq. (2), the $H_{z}(r)$ component in the Bragg fiber core is simply $H_{z}(r)=\mathcal{C}_{T E} J_{0}\left(k_{c o}^{1} r\right)$ and the other two components are ${ }^{1}$

$$
E_{\theta}=-i \frac{\omega \mu_{0}}{k_{c o}^{1}} \mathcal{C}_{T E} J_{0}^{\prime}\left(k_{c o}^{1} r\right), H_{r}=\frac{i \beta}{k_{c o}^{1}} \mathcal{C}_{T E} J_{0}^{\prime}\left(k_{c o}^{1} r\right) .
$$

From these expressions for $E_{\theta}$ and $H_{r}$, we find the power flux along the $z$ direction in the low index core:

$$
P_{z}^{T E}=\left|\mathcal{C}_{T E}\right|^{2} \frac{\pi \omega \mu_{0} \beta}{\left(k_{c o}^{1}\right)^{2}} \int_{0}^{\rho_{c o}^{1}} d r r\left[J_{0}^{\prime}\left(k_{c o}^{1} r\right)\right]^{2}
$$

If the Bragg fiber consists of an infinite number of cladding pairs, the asymptotic fields in the $(N+1)$ th cladding pair can be extracted from Eq. (5)

$$
H_{z}=\frac{f_{T E}}{\sqrt{k_{c l}^{1} r}}\left[c_{N+1} e^{i k_{c l}^{1}\left(r-\rho_{c l}^{N+1}\right)}+d_{N+1} e^{-i k_{c l}^{1}\left(r-\rho_{c l}^{N+1}\right)}\right]
$$




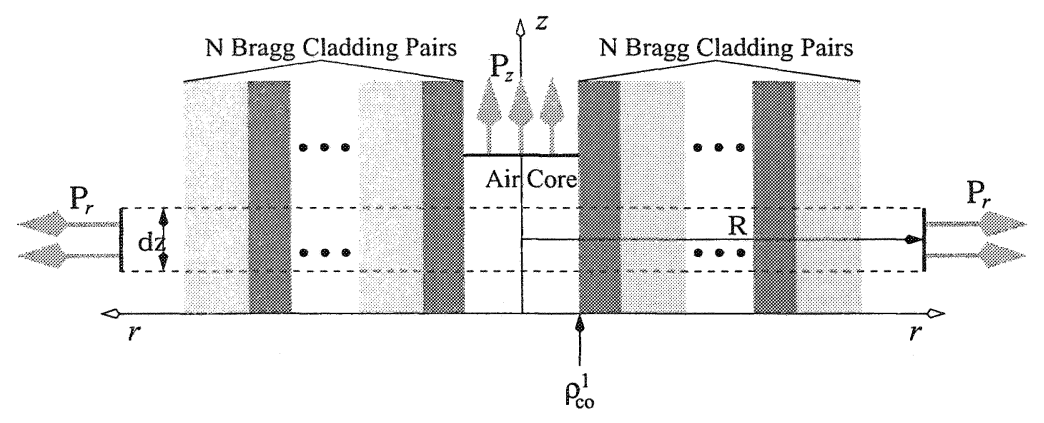

Figure 3. The radiation loss of an air core Bragg fiber with $\mathrm{N}$ cladding pairs. $P_{z}$ is the power flux inside the air core. $P_{r}$ represents the outgoing radiation power flux through a cylindrical surface with radius $R$ and height $d z$.

$$
E_{\theta}=\frac{\omega \mu_{0}}{k_{c l}^{1}} \frac{f_{T E}}{\sqrt{k_{c l}^{1} r}}\left[c_{N+1} e^{i k_{c l}^{1}\left(r-\rho_{c l}^{N+1}\right)}-d_{N+1} e^{-i k_{c l}^{1}\left(r-\rho_{c l}^{N+1}\right)}\right] .
$$

In the above expressions, we notice that the fields consist of two components: an outgoing wave with amplitude proportional to $c_{N+1}$, and an incoming wave with amplitude proportional to $d_{N+1}$. It can be shown that the two components are of equal value and cancel each other such that the Bragg fiber has no net radial power flux. This, however, is directly due to the assumption of infinite cladding pairs. In fact, we can regard the incoming component in the $(N+1)$ th cladding pair as due to the reflection by the remaining Bragg cladding. Therefore, it is reasonable to assume that if the Bragg fiber has only $N$ cladding pairs, the radiation field outside the cladding structure can be well approximated by the outgoing component of Eq. (42) and Eq. (43) (i.e., we replace $d_{N+1}$ with 0 ). To calculate the radial power flow, let us consider a cylindrical surface with radius $\mathrm{R}$ and height $d z$ that encloses the Bragg fiber, as shown in Fig. 3. Using Eq. (42) and Eq. (43) and taking $d_{N+1}=0$, we find the radial power flux through this surface to be

$$
P_{r}^{T E}=\frac{\pi \omega \mu_{0}}{\left(k_{c l}^{1}\right)^{2}}\left|f_{T E}\right|^{2}\left|c_{N+1}\right|^{2} d z .
$$

For TE modes propagating along the $z$ direction of the Bragg fiber, with the presence of radiation loss, the optical power decays as $\exp \left(-\alpha_{T E} z\right)$, where the parameter $\alpha_{T E}$ is the radiation loss constant. From the definitions of $P_{z}^{T E}$ and $P_{r}^{T E}$, we can identify $\alpha_{T E}$ as

$$
\alpha_{T E}=\frac{P_{r}^{T E}}{P_{z}^{T E} d z}=\frac{1}{\beta}\left(\frac{k_{c o}^{1}}{k_{c l}^{1}}\right)^{2}\left|\frac{B_{T E}}{\lambda_{T E}-A_{T E}+B_{T E}}\right|^{2}\left|\lambda_{T E}\right|^{2 N} \frac{\left[J_{0}\left(k_{c o}^{1} \rho_{c o}^{1}\right)\right]^{2} k_{c l}^{1} \rho_{c l}^{1}}{\int_{0}^{\rho_{c o}^{1}} d r r\left[J_{0}^{\prime}\left(k_{c o}^{1} r\right)\right]^{2}} .
$$

For TM modes, we can follow the same procedure to obtain the radiation loss constant $\alpha_{T M}$. First, we find the power flux in $z$ direction within the low index core as

$$
P_{z}^{T M}=\left|\mathcal{A}_{T M}\right|^{2} \frac{\pi \omega \epsilon_{0}\left(n_{c o}^{1}\right)^{2} \beta}{\left(k_{c o}^{1}\right)^{2}} \int_{0}^{\rho_{c o}^{1}} d r r\left[J_{0}^{\prime}\left(k_{c o}^{1} r\right)\right]^{2} .
$$

Similarly, the outgoing radial power flux outside the $N$ th cladding pairs can also be identified from Eq. (5) as

$$
P_{r}^{T M}=\frac{\pi \omega \epsilon_{0}\left(n_{c l}^{1}\right)^{2}}{\left(k_{c l}^{1}\right)^{2}}\left|f_{T M}\right|^{2}\left|a_{N+1}\right|^{2} d z
$$

which gives the radiation loss constant to be

$$
\alpha_{T M}=\frac{P_{r}^{T M}}{P_{z}^{T M} d z}=\frac{1}{\beta}\left(\frac{n_{c l}^{1} k_{c o}^{1}}{n_{c o}^{1} k_{c l}^{1}}\right)^{2}\left|\frac{B_{T M}}{\lambda_{T M}-A_{T M}+B_{T M}}\right|^{2}\left|\lambda_{T M}\right|^{2 N} \frac{\left[J_{0}\left(k_{c o}^{1} \rho_{c o}^{1}\right)\right]^{2} k_{c l}^{1} \rho_{c l}^{1}}{\int_{0}^{\rho_{c o}^{1}} d r r\left[J_{0}^{\prime}\left(k_{c o}^{1} r\right)\right]^{2}} .
$$

We introduce a new parameter $x=k_{c o}^{1} \rho_{c o}^{1}$. For the fiber structure shown in Fig. $3, \rho_{c l}^{1}$ is the same as $\rho_{c o}^{1}$, which gives

$$
\frac{\left[J_{0}\left(k_{c o}^{1} \rho_{c o}^{1}\right)\right]^{2} k_{c l}^{1} \rho_{c l}^{1}}{\int_{0}^{\rho_{c o}^{1}} d r r\left[J_{0}^{\prime}\left(k_{c o}^{1} r\right)\right]^{2}}=k_{c o}^{1} k_{c l}^{1} \frac{x\left[J_{0}(x)\right]^{2}}{\int_{0}^{x} d u u\left[J_{1}(u)\right]^{2}}
$$


where we have applied $J_{0}^{\prime}(x)=-J_{1}(x) .{ }^{13} \quad$ For an order of magnitude estimate, we can simply choose $x=3.8317$, the first zero point of $J_{1}(x)$, and the integral $\int_{0}^{x} d u u\left[J_{1}(u)\right]^{2}$ becomes $x^{2}\left[J_{2}(x)\right]^{2} / 2 .{ }^{13}$ Combining these results, we find

$$
\frac{\left[J_{0}\left(k_{c o}^{1} \rho_{c o}^{1}\right)\right]^{2} k_{c l}^{1} \rho_{c l}^{1}}{\int_{0}^{\rho_{c o}^{1}} d r r\left[J_{0}^{\prime}\left(k_{c o}^{1} r\right)\right]^{2}} \approx 0.522 k_{c o}^{1} k_{c l}^{1} .
$$

As can be seen from Eq. (11) to Eq. (16), $A_{T E}, B_{T E}, \lambda_{T E}, A_{T M}, B_{T M}$, and $\lambda_{T M}$ have the same order of magnitude. Therefore, in our estimation of radiation loss, we take the values of $B_{T E} /\left(\lambda_{T E}-A_{T E}+B_{T E}\right)$ and $B_{T M} /\left(\lambda_{T M}-\right.$ $\left.A_{T M}+B_{T M}\right)$ to be 1. Combining these approximations with Eq. (50), we find Eq. (45) and Eq. (48) become

$$
\alpha_{T E}=0.522 \frac{\left(k_{c o}^{1}\right)^{3}}{\beta k_{c l}^{1}}\left|\lambda_{T E}\right|^{2 N}, \alpha_{T M}=0.522\left(\frac{n_{c l}^{1}}{n_{c o}^{1}}\right)^{2} \frac{\left(k_{c o}^{1}\right)^{3}}{\beta k_{c l}^{1}}\left|\lambda_{T M}\right|^{2 N}
$$

These two expressions can be further simplified by taking $n_{c o}^{1}=1$ (air core), $\lambda=2 \pi c / \omega=1.55 \mu m$, and assuming $\beta=k_{c o}^{1}=\omega / \sqrt{2} c, k_{c l}^{1}=n_{c l}^{1} \omega / c$ :

$$
\alpha_{T E}(\mathrm{~dB} / \mathrm{km})=4.6 \times 10^{9} \frac{1}{n_{c l}^{1}}\left|\lambda_{T E}\right|^{2 N}, \quad \alpha_{T M}(\mathrm{~dB} / \mathrm{km})=4.6 \times 10^{9} n_{c l}^{1}\left|\lambda_{T M}\right|^{2 N},
$$

where the unit for radiation loss has been converted to $\mathrm{dB} / \mathrm{km}$. Many assumptions are made to simplify Eq. (45) and Eq. (48) into (52). It is worthwhile to see how we can justify the simplified results from an intuitive point of view. Without the Bragg cladding, the light confinement can only be achieved on the order of the wavelength, which means that the radiation loss constant must be of the order of $\mathrm{dB} / \mu m=10^{9} \mathrm{~dB} / \mathrm{km}$. With the presence of Bragg cladding, the light amplitude reduction due to each cladding pair is $\lambda_{T E}$ for TE modes and $\lambda_{T M}$ for TM modes. Therefore, the radiation loss for a fiber with $\mathrm{N}$ Bragg cladding pairs should be of the order of $\left(\lambda_{T E}\right)^{2 N} \times 10^{9} \mathrm{~dB} / \mathrm{km}$ for TE modes and $\left(\lambda_{T M}\right)^{2 N} \times 10^{9} \mathrm{~dB} / \mathrm{km}$ for TM modes.

The values of $\lambda_{T E}$ and $\lambda_{T M}$ also have complicated dependence on $\beta, n_{c l}^{1}, l_{c l}^{1}, n_{c l}^{2}$ and $l_{c l}^{2}$. However, when the cladding layers form quarter wave stack (i.e., $k_{c l}^{1} l_{c l}^{1}=k_{c l}^{2} l_{c l}^{2}=\pi / 2$ ) such that light is optimally confined, the expressions for $\left|\lambda_{T E}\right|$ and $\left|\lambda_{T M}\right|$ take simpler forms:

$$
\left|\lambda_{T E}\right|=\min \left(\frac{k_{c l}^{2}}{k_{c l}^{1}}, \frac{k_{c l}^{1}}{k_{c l}^{2}}\right),\left|\lambda_{T M}\right|=\min \left[\left(\frac{n_{c l}^{2}}{n_{c l}^{1}}\right)^{2} \frac{k_{c l}^{1}}{k_{c l}^{2}},\left(\frac{n_{c l}^{1}}{n_{c l}^{2}}\right)^{2} \frac{k_{c l}^{2}}{k_{c l}^{1}}\right] .
$$

We choose cladding layer II to be the low index medium with $n_{c l}^{2}=1.5$, typical of silica glass and polymers. With this value, it can be shown that for $0<\beta<\omega / c$ the minimum value of $\left|\lambda_{T E}\right|$ is $\sqrt{\left[\left(n_{c l}^{2}\right)^{2}-1\right] /\left[\left(n_{c l}^{1}\right)^{2}-1\right]}$, and the minimum value of $\left|\lambda_{T M}\right|$ is $n_{c l}^{2} / n_{c l}^{1}$. Substituting them into Eq. (52), we find the minimum number of Bragg layer pairs required to achieve $0.2 \mathrm{~dB} / \mathrm{km}$ radiation loss is

$$
N_{T E}=\frac{23.9-\ln \left(n_{c l}^{1}\right)}{\ln \left[\left(n_{c l}^{1}\right)^{2}-1\right]-\ln \left[\left(n_{c l}^{2}\right)^{2}-1\right]}, \quad N_{T M}=\frac{23.9+\ln \left(n_{c l}^{1}\right)}{2\left[\ln \left(n_{c l}^{1}\right)-\ln \left(n_{c l}^{2}\right)\right]}
$$

for TE and TM modes, respectively.

We plot Eq. (54) in Fig. 4. The top figure corresponds to the case of weak index contrast. For $\Delta n$ less than 0.01 , it takes 1000 or more cladding pairs to reach $0.2 \mathrm{~dB} / \mathrm{km}$. Fabricating such large number of cladding pairs is likely to be very difficult in practice. For $\Delta n$ between 0.1 and 1, we find that it takes less than 200 cladding pairs to reduce the radiation loss of $T E$ and $T M$ modes to $0.2 \mathrm{~dB} / \mathrm{km}$. We notice that this index contrast range corresponds to what can be achieved in air core PBG fiber. ${ }^{6}$ Of course, the light confinement in PBG fibers is achieved through two-dimensional Bragg reflection rather than one-dimensional Bragg reflection. However, if we take an effective index approach and approximate the 2D air hole patterns as alternating layers of concentric dielectric layers with high and low refractive index, the index contrast between the effective refractive indices should fall within the range of 0.1 to 1. Thus for air core PBG fibers, $0.2 \mathrm{~dB} / \mathrm{km}$ propagation loss can be achieved with 200 or less air hole layers. The bottom figure in Fig. 4 corresponds to the case of large index contrast. We notice that for $\Delta n$ between 1 and 3 $\left(2.5<n_{c l}^{1}<4.5\right), 25$ pairs may suffice to guide TE and TM modes with less than $0.2 \mathrm{~dB} / \mathrm{km}$ radiation loss.

We have only discussed radiation loss for TE and TM modes so far. According to the discussions in Sec. 2.3, modes with $m \neq 0$ are mixtures of TE and TM components in the Bragg cladding layers. Therefore, their radiation 

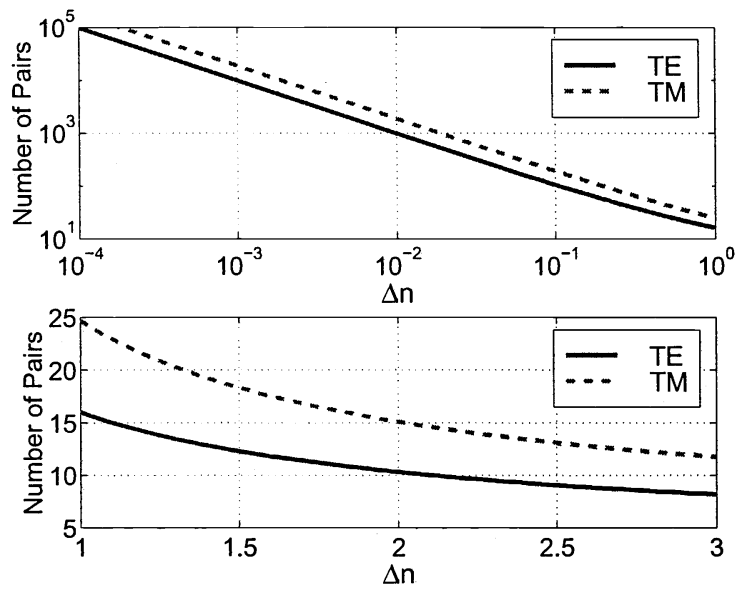

Figure 4. The number of Bragg cladding pairs necessary to achieve $0.2 \mathrm{~dB} / \mathrm{km}$ radiation loss. $\Delta n$ is the index contrast between the two cladding dielectric media $n_{c l}^{1}-n_{c l}^{2}$, with $n_{c l}^{2}=1.5$. The solid line gives the minimum Bragg pairs for TE modes to $0.2 \mathrm{~dB} / \mathrm{km}$, while the dash line gives the corresponding quantity for the TM modes.

loss is determined by the TM component, since TM component is less confined and suffers more radiation loss compared with TE component, as can be seen from Fig. 4.

Since we use the smallest possible values for $\left|\lambda_{T E}\right|$ and $\left|\lambda_{T M}\right|$ in deriving Eq. (54), our results in Fig. 4 give the minimum number of Bragg pairs needed to achieve $0.2 \mathrm{~dB} / \mathrm{km}$ radiation loss and should serve as an order of magnitude estimate. Obtaining a better estimate of radiation loss requires values of $\beta, n_{c l}^{1}, l_{c l}^{1}, n_{c l}^{2}$, and $l_{c l}^{2}$. Once they are known, we can find $\lambda_{T E}$ and $\lambda_{T M}$ from Eq. (15) and (16), and substitute them into Eq. (51) for $\alpha_{T E}$ and $\alpha_{T M}$.

One notable exception to the above estimate is worth mentioning. In an air core fiber, if $\beta$ is only slightly less than $\omega / c$, the value of $k_{c o}^{1}$ can be very close to zero (whereas in deriving Eq. (54) we use $\beta=k_{c o}^{1}=\omega / \sqrt{2} c$ ). According to Eq. (45) and Eq. (48), a small $k_{c o}^{1}$ can greatly reduce the radiation loss. ${ }^{2}$ Therefore, when $\beta$ becomes very close to $\omega / c$, it may be necessary to use Eq. (45) and Eq. (48) to obtain accurate results of radiation loss.

\section{BRAGG FIBER DISPERSION}

Having developed the asymptotic formalism in the previous section, we shall apply it to study the dispersion of a Bragg fiber. We choose to study an air core $\left(n_{c o}^{1}=1.0\right)$ Bragg fiber with cladding parameters as follows: $n_{c l}^{1}=4.6$, $l_{c l}^{1}=0.25 \Lambda, n_{c l}^{2}=1.5$ and $l_{c l}^{2}=0.75 \Lambda$, where the parameters are defined in Fig. 2 and $\Lambda=l_{c l}^{1}+l_{c l}^{2}$ is the total thickness of a Bragg cladding pair. We choose the air core radius to be $\rho_{c o}^{1}=1.0 \Lambda$. In the asymptotic calculations, the core region consists of 5 concentric dielectric layers. Using the notations in Fig. 2, we explicitly write out the core region parameters as $n_{c o}^{1}=1.0, n_{c o}^{2}=n_{c o}^{4}=4.6, n_{c o}^{3}=n_{c o}^{5}=1.5, l_{c o}^{1}=1.0 \Lambda, l_{c o}^{2}=l_{c o}^{4}=0.25 \Lambda$, and $l_{c o}^{3}=l_{c o}^{5}=0.75 \Lambda$. In 2D FDTD calculations, we choose $\Lambda=24$ computational cells and use 3 cladding pairs around the air core to define the Bragg fiber. In Fig. 4, we find that for index contrast we have chosen, 10 cladding pairs are enough to reduce the radiation loss to approximately $0.2 \mathrm{~dB} / \mathrm{km}$. Not surprisingly, 3 cladding pairs should give us well defined guided modes.

Both the asymptotic results and the FDTD results are shown in Fig. (5a), where we plot the effective index $n_{\text {eff }}=\beta c / \omega$ as a function of $\omega$, and $\omega$ as a function of propagation constant $\beta$. Within the frequency range shown in Fig. 5, both the asymptotic analysis and FDTD calculations show that the Bragg fiber supports a guided mode with $m=1$ propagating in the air core. In Fig. (5a), the two approaches agree well with each other, while the small discrepancy can be attributed to the discretization error in the FDTD algorithm. In fact, if we consider that only 6 computational cells are used for $l_{c l}^{1}$, the agreement between the asymptotic approach and FDTD approach is quite impressive. In Fig. (5b), we show the distribution of the $H_{z}$ field obtained from FDTD calculation. The frequency and propagation constant of the mode are respectively $\omega=0.291(2 \pi c / \Lambda)$ and $\beta=0.143(2 \pi / \Lambda)$. Fig. (5b) clearly 

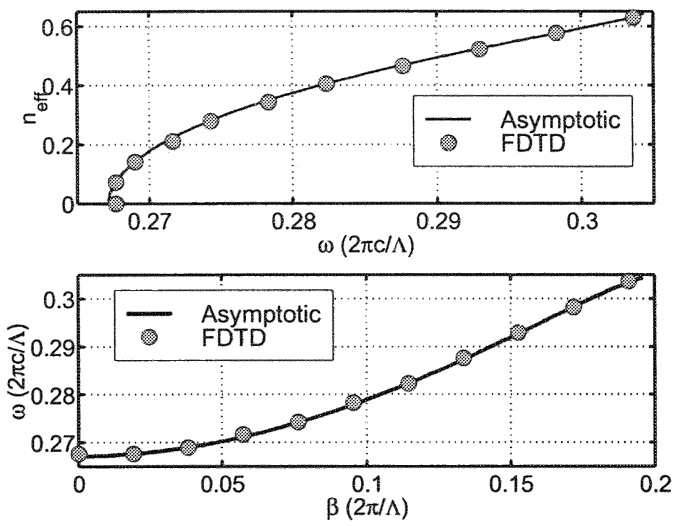

(a)

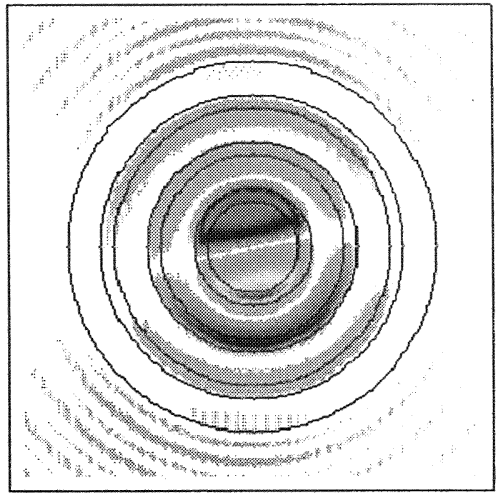

(b)

Figure 5. (a) The dispersion of an air core Bragg fiber with a $m=1$ mode. The Bragg fiber parameters are: $n_{c o}^{1}=1.0, \rho_{c o}^{1}=1.0 \Lambda, n_{c l}^{1}=4.6, l_{c l}^{1}=0.25 \Lambda, n_{c l}^{2}=1.5$ and $l_{c l}^{2}=0.75 \Lambda$. The solid line is from the asymptotic analysis, while the dots represent the 2D FDTD results. The effective indices $n_{\text {eff }}$ is defined as $\beta c / \omega$. (b) The $H_{z}$ field distribution of the guided $m=1$ Bragg fiber mode at $\omega=0.291(2 \pi c / \Lambda)$ and $\beta=0.143(2 \pi / \Lambda)$.

shows that the guided mode has an azimuthal number $m=1$ and most of the field is concentrated within the air core and the first cladding layer. The radiation field outside of the Bragg cladding can also be seen in Fig. (5b).

To find the field distribution using the asymptotic approach, we must first obtain the propagation constant using Eq. (33). Substituting the result into Eq. (34) and Eq. (35), we obtain the modal amplitude coefficients in the first layer of the cladding region (i.e., $f_{T M}$ and $f_{T E}$ ) and those in the center air core (i.e., $\mathcal{A}_{T M}$ and $\mathcal{C}_{T E}$ ), respectively. Then the cladding fields are easily found from Eq. (5) and Eq. (6), while the core fields are obtained by applying Eq. (2) and Eq. (3) repeatedly. We apply this algorithm to study the field distribution of the guided Bragg fiber mode at $\omega=0.286(2 \pi c / \Lambda)$. Using a core region of 5 layers, we find the propagation constant to be $\beta=0.128(2 \pi / \Lambda)$. The field distribution given by this asymptotic approach is represented by the solid lines in Fig. 6 .
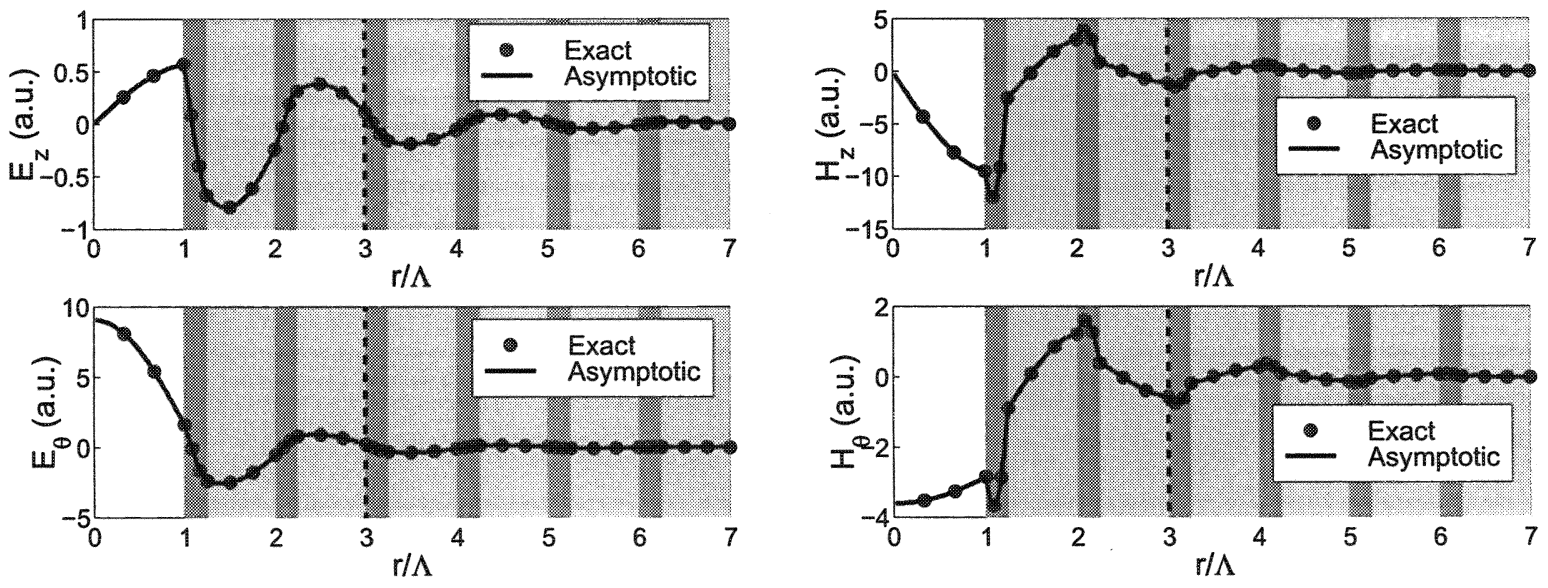

Figure 6. The electromagnetic field distribution of the guided Brag fiber mode at $\omega=0.286(2 \pi c / \Lambda)$. The interface between the core region and cladding region is indicated by dash line. The exact solutions are obtained using Eq. (2) and (3) only. The asymptotic solutions are obtained using Eq. (2) and Eq. (3) in the core region, and Eq. (5) and (6) in the cladding region.

Having obtained the field distribution using the asymptotic algorithm, naturally we would like to know how 
accurate the asymptotic approximation works without having to check asymptotic results using either the orginal algorithm by Yeh et al. or the FDTD method. We notice that the essence of the whole asymptotic algorithm is that the field distribution in the cladding region can be well described in the asymptotic limit. As long as this condition is satisfied, the asymptotic approach should provide a satisfactory description of the guided mode. To check the accuracy of the asymptotic approximation in the cladding region, we can calculate the exact field distribution in the cladding region by repeatedly applying Eq. (2) and (3). We use "exact solution" to denote results obtained this way. In other word, to find the "exact solution," we still need to find $\mathcal{A}_{T M}$ and $\mathcal{C}_{T E}$ using Eq. (33), (34) and (35), as described in the previous paragraph. The only difference between the "exact solution" and the asymptotic solution is that for the "exact solution," the field distribution in the entire Bragg fiber is obtained from Eq. (2) and (3). Consequently, within the core region, the "exact solution" and the asymptotic solution are the same. In the cladding region, the two solutions differ from each other, and their difference indicates how well the asymptotic approximation works. In Fig. 6, the "exact solutions" are represented by the dots. As expected, the "exact solution" and the asymptotic solution are the same within the core region. However, even in the cladding region, the difference between the two solutions are very small. Thus we can conclude that the asymptotic algorithm gives an accurate description of the field distribution of the guided mode.

It should be mentioned that our asymptotic algorithm can be arbitrarily precise by incorporating more and more dielectric layers into the core region. More specifically, if we use a superscript $N$ to denote the asymptotic results obtained using an inner core region consisting of $N$ dielectric layers, the results should converge as a function of $N$ to the exact solutions. Thus the asymptotic results can be arbitrarily precise.

\section{COAXIAL FIBER DISPERSION}

It is well known that the fundamental TEM mode of a metallic coaxial cable has no polarization effect or any modal dispersion, which make it very attractive for transmitting electromagnetic signals. The problem is that metals are very lossy in the optical range. Recently Ibanescu et al. drew an analogy between the metallic coaxial cables and dielectric coaxial fibers, and proposed to use dielectric coaxial fibers in optical communications. ${ }^{7}$ There are, however, several important problems to be solved before coaxial fibers can find wide applications in optical telecommunication. Firstly, it is critical that the coaxial fiber mode has small dispersion within the entire telecom frequency window instead of at a single point. Secondly, we should keep in mind that the analogy between omnidirectional mirrors and high refractive index materials with metals is not perfect. For example, if the outer cladding of a metallic coaxial cable is taken out, the center metal rod does not support lossless propagating mode. Yet if we take away the Bragg cladding of the coaxial fiber, the center high index dielectric rod resembles an optical fiber and supports at least one propagating mode. In this section, we apply the asymptotic matrix theory to address the aforementioned problems.

As in Bragg fibers, each guided coaxial fiber mode can be classified according to its propagation constant $\beta$ (momentum in the $z$ direction) and angular momentum $m$. Using the asymptotic method, we analyze one of the coaxial fibers studied by Ibanescu et al. $^{7}$ For the high index medium of the coaxial fiber cladding, we choose $n_{c l}^{1}=4.6$ and $l_{c l}^{1}=(1 / 3) \Lambda$, whereas for the low index cladding medium we have $n_{c l}^{2}=1.6$ and $l_{c l}^{2}=(2 / 3) \Lambda$. $\Lambda$ is the total thickness of the Bragg cladding pair. The parameters of the Bragg stack are chosen such that it forms an omnidirectional reflector. ${ }^{7}$ The refractive index and radius of the center core are respectively $n_{c o}=4.6$ and $l_{c o}=0.4 \Lambda$. The refractive index and thickness of the coaxial region are respectively $n_{\text {coax }}=1$ and $l_{\text {coax }}=1.0 \Lambda$. We use four core layers in our asymptotic calculations and normalize the results with respect to $\Lambda$. The asymptotic results are shown in Fig. (7a) as thick solid lines. We also use the 2D FDTD algorithm to verify the validity of our asymptotic calculations. The FDTD results are shown in Fig. (7a) as dots. The shaded region in Fig. (7a) corresponds to the TM cladding modes that can propagate in the cylindrical omnidirectional reflector.

In Fig. (7a), the asymptotic analysis gives us four photonic bands, a TE band $(m=0)$, a TM band $(m=0)$, and two $m=1$ bands. The asymptotic results agree well with FDTD calculations. We point out that the TE band was missed in the results obtained by Ibanescu et al. ${ }^{7}$ We notice that the asymptotic results for the TM band and two $m=1$ bands are confined within the region of TM gap. This is simply due to the fact that all three bands contain TM components and that the TM components must decay in the Bragg cladding to define guided coaxial fiber modes. The TE band, on the other hand, does not contain any TM component and asymptotic analysis gives us guided TE modes up to the light line in cladding medium II $\left(\beta=n_{c l}^{2} \omega / c\right)$. The excellent agreement between the asymptotic analysis and FDTD calculations demonstrates the validity of asymptotic approach. 
As mentioned before, if we take out the Bragg cladding, the center dielectric core becomes a conventional optical fiber whose modal dispersion is well known. ${ }^{15}$ The dispersion of these conventional fiber modes was plotted in Fig. (7a) as dash lines. Comparing the conventional fiber modes with the FDTD calculations of the full coaxial fiber, we find excellent correspondence between the two for the region below the air light line $\beta=\omega / c$. This strongly suggests that once the guided coaxial fiber modes pass through the air light line, the main confining mechanism is actually provided by the center high index core. To see this point more clearly, we show two FDTD calculations of field distribution of the lower $m=1$ band. The $m=1$ mode in Fig. (7b) has $\beta=0.153(2 \pi / \Lambda), \omega=0.187(2 \pi c / \Lambda)$ and belongs to the TM bandgap above the air light line. For any modes above light line, guiding cannot be achieved through total internal reflection and therefore in Fig. (7b) we observe a substantial field distribution in both the coaxial region (air) and the Bragg cladding. The $m=1$ mode in Fig. (7c) has $\beta=0.611(2 \pi / \Lambda), \omega=0.229(2 \pi c / \Lambda)$ and is clearly below the air light line. As expected, the guided coaxial fiber mode becomes essentially the HE mode of a conventional fiber, with optical fields concentrated in the center dielectric core and only a negligible amount in the Bragg cladding.

Not only does the total internal reflection play a significant role in the modal dispersion of the guided coaxial fiber below the light line, it also must be taken into account in determining the frequency window of single mode operation. The lower single mode window, as shown in Fig. (7a), simply contains all the TM modes below the cutoff frequency of the lower $m=1$ band. However, finding the higher single mode window is more trickier. As the lower $m=1$ band enters the shaded region in Fig. (7a), the TM field component loses confinement in the Bragg cladding and the $m=1$ band is no longer a well defined guided mode. Thus the second single TM mode window in Fig. (7a) begins at the lower intersection of the $m=1$ band and the TM gap, and ends at the smallest of the following frequencies: the cutoff frequency of the higher $m=1$ band, the cutoff frequency of the TE band, and the higher intersection point of the lower $m=1$ band and the TM gap where the lower $m=1$ band enters again into the TM

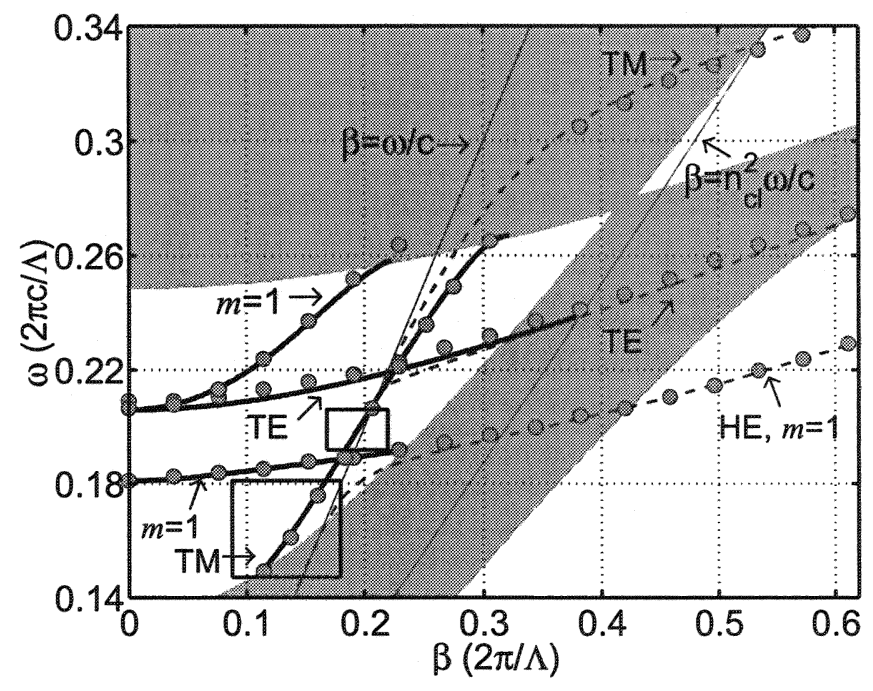

(a)

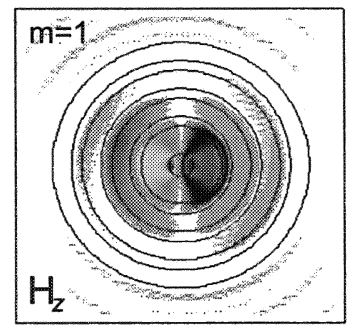

(b)

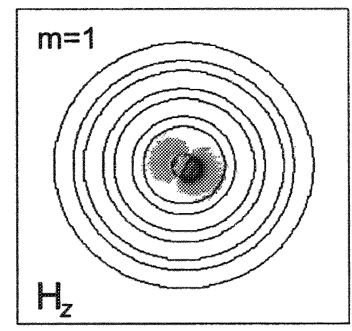

(c)

Figure 7. (a) Dispersion of the coaxial fiber. The points in the shaded region indicate the existence of propagating TM cladding modes in the omnidirectional reflector. The thick solid lines are results obtained from asymptotic analysis. The dots represent the 2D FDTD results. The light lines in air $(\beta=\omega / c)$ and in the low index medium of the Bragg cladding $\left(\beta=n_{c l}^{2} \omega / c\right)$ are also shown. If the omnidirectional cladding is taken away, the center core of the coaxial fiber resembles a conventional optical fiber and supports three guided modes: HE, TE and TM modes. Their dispersions are calculated using the formulae for conventional optical fibers and are shown as dash lines. The single mode windows for the TM band are illustrated in the figure as two boxes. The $H_{z}$ field distributions of the lower $m=1$ band are shown in (b) and (c). In (b), the guided mode has $\beta=0.153(2 \pi / \Lambda)$ and $\omega=0.187(2 \pi c / \Lambda)$. In (b), the guided mode has $\beta=0.611(2 \pi / \Lambda)$ and $\omega=0.229(2 \pi c / \Lambda)$. 
gap. The two single frequency windows are shown in Fig. (7a) as two boxes. Within the higher single frequency window, the $m=1$ quasi-band, even though not well confined, can still have low loss due to total internal reflection in the coaxial air region. In fact, that is exactly why FDTD algorithm can give us $m=1$ and TM band outside of the TM gap. To study the influence of the $m=1$ quasi-band on the single mode operation of TM band, however, is beyond the scope of this section.
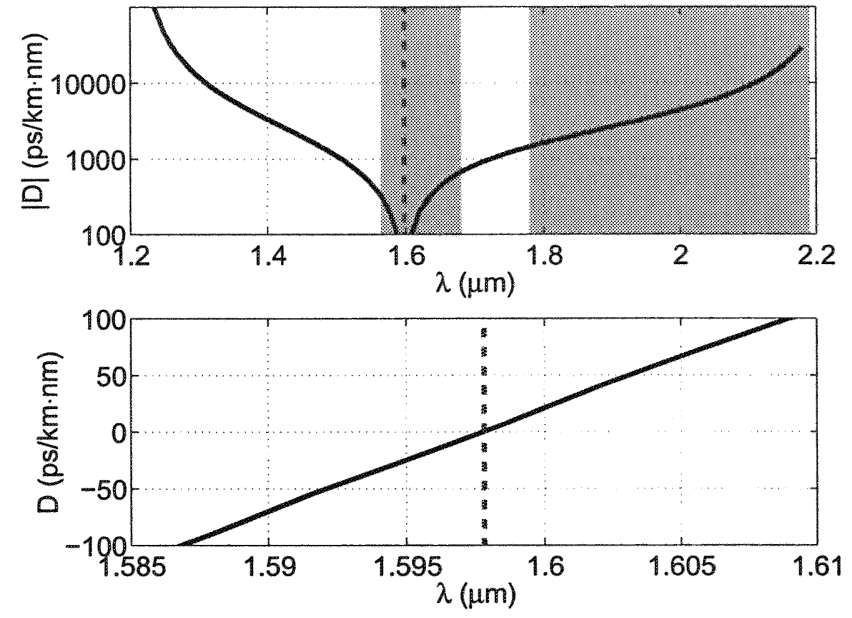

(a)
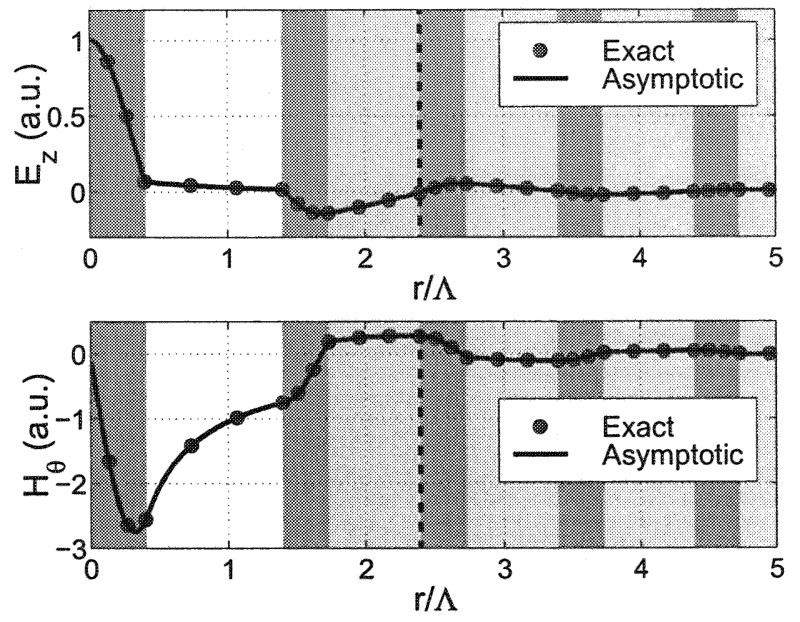

(b)

Figure 8. (a) Dispersion parameter D of the coaxial fiber TM band. At $\lambda=1.598 \mu m$ [or $\omega=0.202(2 \pi c / \Lambda)$ ], the dispersion parameter $\mathrm{D}$ becomes zero, which is shown as the dash line. In the upper diagram, the absolute values of $\mathrm{D}$ are show in a log scale. The two single mode windows in Fig. 7 are shown as shaded regions. To the left of the dash line, $\mathrm{D}$ is negative, whereas $\mathrm{D}$ is positive to the left of the dash line. In the lower diagram, $\mathrm{D}$ is shown in the linear scale. (b) The $E_{z}$ and $H_{\theta}$ fields of the TM coaxial fiber mode at $\omega=0.202(2 \pi c / \Lambda)$. The unshaded, light, and dark regions respectively represent air $\left(n_{c o a x}=1\right)$, low index dielectric medium $\left(n_{c l}^{2}=1.6\right)$ and high index dielectric medium $\left(n_{c l}^{1}=4.6\right)$. The units for electric field and magnetic field are chosen such that $\epsilon_{0}=1$ and $\mu_{0}=1$.

For the long distance communication fibers, the dispersion parameter D, which is defined as $-\frac{2 \pi c}{\lambda^{2}} \frac{d^{2} \beta}{d \omega^{2}},{ }^{15}$ should remain small within the entire telecommunication window. In Fig. (8a), we show the dispersion parameter D calculated from the asymptotic results. The wavelength $\lambda$ is normalized such that the TM band crosses air light line $\beta=\omega / c$ at $1.55 \mu \mathrm{m}$. The two single frequency windows are identified in Fig. (8) as shaded region. We immediately notice that the dispersion parameter $\mathrm{D}$ takes very large value at most frequencies and can be both positive and negative. Around $1.6 \mu m[\omega=0.202(2 \pi c / \Lambda)]$, D crosses the point of zero dispersion but remains small only within a very small frequency range. Ibanescu et al. predicted a point of zero dispersion. ${ }^{7}$ Our results in Fig. (8a) confirm their prediction, yet at the same time, point out an important problem: The frequency window of small $\mathrm{D}$ is too narrow for optical signal transmission.

In Fig. (8b), we show $E_{z}$ and $H_{\theta}$ components of the TM mode at the zero dispersion frequency $\omega=0.202(2 \pi c / \Lambda)$. Since the magnetic field of a TM mode contains only $H_{\theta}$ component, from Fig. (8b) it is obvious that there is substantial presence of electromagnetic field in the high index core and the optical intensity in the high index core is comparable to that in the air coaxial region. As a result, using this coaxial fiber mode to guide light does not provide much benefit in terms of reducing material absorption and nonlinear effects. This also illustrates that the analogy between dielectric coaxial fibers and metallic coaxial cables is not perfect. Turning our attention to the cladding field, we find that the field strength in the first cladding pairs, even though relatively small, is not negligible. In fact, the fields in the first Bragg pairs cannot be neglected, since optical fields must penetrate at least one cladding pair to experience Bragg confinement. This also explains the large modal dispersion we find in Fig. (8a), since any guided coaxial fiber mode must "feel" several different dielectric media: the high index core, air in the coaxial region, the high index cladding, and the low index cladding. In contrast, for conventional optical fibers, the guided modes are defined by the silica core and cladding whose index difference is generally less than 0.01 . 
Summarizing this section, we find that both Bragg reflection and total internal reflection play important roles in determining modal dispersion of the coaxial fiber. The analogy between dielectric coaxial fibers and metallic coaxial cable are not entirely accurate, and there is substantial amount of optical fields in the high index core and the Bragg cladding. As a result, guided coaxial fiber mode generally have large dispersion.

\section{ACKNOWLEDGMENTS}

This research was sponsored by the Office of Naval Research, whose support is gratefully acknowledged.

\section{REFERENCES}

1. P. Yeh, A. Yariv, and E. Marom, "Theory of Bragg fiber," J. Opt. Soc. Amer., vol. 68, pp. 1196-1201, 1978.

2. N. J. Doran, and K. J. Blow, "Cylindrical Bragg fibers: a design and feasibility study for optical communications," J. Lightwave Technol., vol. LT-1, pp. 588-590, 1983.

3. J. C. Knight, T. A. Birks, P. St. J. Russell, and D. M. Atkin, "All-silica single-mode optical fiber with photonic crystal cladding," Opt. Lett., vol. 21, pp. 1547-1549, 1996.

4. Y. Fink, D. J. Ripin, S. Fan, C. Chen, J. D. Joannopoulos, and E. L. Thomas, "Guiding optical light in air using an all-dielectric structure," J. Lightwave Technol., vol. 17, pp. 2039-2041, 1999.

5. J. C. Knight, J. Broeng, T. A. Birks, and P. St. J. Russell, "Photonic band gap guidance in optical fibers," Science, vol. 282, pp. 1476-1478, 1998.

6. R. F. Cregan, B. J. Mangan, J. C. Knight, T. A. Birks, P. St. J. Russell, P. J. Roberts, and D. C. Allan, "Single-mode photonic band gap guidance of light in air," Science, vol. 285, pp. 1537-1539, 1999.

7. M. Ibanescu, Y. Fink, S. Fan, E. L. Thomas, and J. D. Joannopoulos, "An all-dielectric coaxial waveguide," Science, vol. 289, pp. 415-419, 2000.

8. Y. Xu, R. K. Lee, and A. Yariv, "Asymptotic analysis of Bragg fibers," Opt. Lett., vol. 25, pp. 1756-1758, 2000.

9. J. N. Winn, Y. Fink, S. Fan, and J. D. Joannopoulos, "Omnidirectional reflections from a one-dimensional photonic crystal," Opt. Lett., vol. 23, pp. 1573-1575, 1998.

10. Y. Fink, J. N. Winn, S. Fan, C. Chen, J. Michel, J. D. Joannopoulos, and E. L. Thomas, "A dielectric omnidirectional reflector," Science, vol. 282, pp. 1679-1682, 1998.

11. D. N. Chigrin, A. V. Lavvrinenko, D. A. Yarotsky, and S. V. Gaponenko, "Observation of total omnidirectional reflection from one-dimensional dielectric lattice," Appl. Phys. A, vol. 68, pp. 25-28, 1999.

12. F. Zepparelli, P. Mezzanotte, F. Alimenti, L. Roselli, R. Sorrentino, G. Tartarini, and P. Bassi, "Rigorous analysis of 3D optical and optoelectronic devices by the compact-2D-FDTD method," Opt. and Quantum Electron., vol. 31, pp. 827-841, 1999.

13. J. Mathews, and R. L. Walker, Mathematical methods of physics, Addison-Wesley, 1970.

14. P. Yeh, and A. Yariv, "Bragg reflection waveguides," Opt. Commun., vol. 19, pp. 427-430, 1976.

15. A. Yariv, Optical electronics in modern communications, Oxford University Press, New York, 1997. 